\title{
REALISTIC MODELS FOR DONORS IN ULTRACOMPACT BINARIES
}

\author{
C. J. Deloye ${ }^{1}$ and L. Bildsten ${ }^{1.2}$
}

\section{RESUMEN}

Presentamos el primer conjunto realista de modelos estelares arbitrariamente degenerados, apropiados para donadores en binarias ultracompactas. Las relaciones masa radio, M-R, de estos objetos son parametrizadas por la entropía y la composición del donador. Enfatizamos que nuestra comprensión de la configuración actual de binarias ultracompactas y sus potenciales trazas evolutivas pueden estar afectadas por este hecho.

\section{ABSTRACT}

We present the first realistic set of arbitrarily degenerate stellar models appropriate for donors in ultracompact binaries. The mass-radius $M-R$ relations for these objects are parameterized by the donor's ent ropy and composition. We highlight how this fact can affect our understanding on the current configuration of ultracompact binaries today and their potential evolutionary tracks.

\section{Key Words: BINARIES: CLOSE - WHITE DWARFS - X-RAYS: BINARIES}

\section{MODELS FOR ARBITRARILY DEGENERATE DONORS}

Ultracompact binaries (UCBs) are masstransferring systems with orbital periods, $P_{\text {orb }}$, below the minimum which binaries with predominantly $\mathrm{H}$ donors can reach $(\approx 70-80 \mathrm{~min})$ (Rappaport, Joss, \& Webbink 1982). The donors in UCBs have evolved (He or C/O) compositions (Nelson, Rappaport, \& Joss 1986; Fedorova \& Ergma 1989; Podsiadlowski, Rappaort, \& Pfahl 2002) and most likely have masses $M_{2} \lesssim 0.1 M_{\text {c., }}$ (e.g.; Markwardt et al. (2002); Galloway et al. (2002)). Although, typically the donors in UCBs are modeled as fully degenerate objects, we expect the donors to have core temperatures, $T_{c}$, between $10^{5}-10^{7} \mathrm{~K}$, depending on the binary's formation channel and the donor's composition (Bildsten 2002; Nelson \& Rappaport 2003; Serenelli, Althaus, Rohrmann, \& Benvenuto 2001; Salaris et al. 2000). This make them far from fully degenerate in most cases and has implications for the current states of UCB systems and their potential evolutionary histories, past and future. In particular, approximating these donors as fully degenerate is not sufficient for understanding the properties of the UCB population.

To address the impact of partial degeneracy on the structure of UCB donors, we have calculated the first realistic set of arbitrarily degenerate models relevant to these objects. A sample of the resulting $M-R$ relations are shown in Figure 1. We emphasize that these relations are not single-valued, but param-

\footnotetext{
${ }^{1}$ Dept. of Physics, UCSB

${ }^{2}$ KITP, UCSB
}

eterized by the donor's entropy and composition. In particular, for Roche Lobe (RL) filling systems at a given $P_{\text {orb }}$, there are multiple combinations of $T_{c}$ and composition for which a RL-filling donor can be found. The binary's configuration and evolutionary path varies with these parameters.

\section{IMPLICATIONS OF MULTI-VALUED $M-R$ RELATIONS FOR ULTRACOMPACT BINARIES}

For example, in the ultracompact accreting millisecond pulsar systems, where we know mass function, we can correlate the orbital inclination with the donor's $T_{r}$ and composition. In particular, the sy'stem XTE J1751-305 (Markwardt et al. 2002), does not admit a fully degenerate donor. Also, the probability of finding a He donor in the systems XTE J0929-314 and XTE J1807-294 (Galloway ot al. 2002: Markwardt et al. 2003a,b), from inclination constraints due to the $T_{c}=0$ solution, is $35 \%$ and $15 \%$, respectively. In general, though, for any given orbital inclination, a combination of donor $T_{c}$ and composition can be found that produces a RL-filling donor.

The evolution of UCBs also depends on the donor's $T_{c}$ and composition. For example, the relation between the mass transfer rate. $i$, and $P_{\text {orb }}$ in UCBs is multiply-valued (Figure 2) along with the $M-R$ relations. At longer $P_{\text {orb }}$, the variation in $\dot{H}$ from $T_{c}$ variations can be 2-3 orders of magnitude. if the donors can be arbitrarily hot. However, a donor evolving adiabatically from short $P_{\text {orb }}$ cannot be arbitrarily hot at longer $P_{\text {orb }}$ (dashed line in Figure 2). Thus some regimes of the $\dot{M}-P_{\text {orb }}$ can only be 


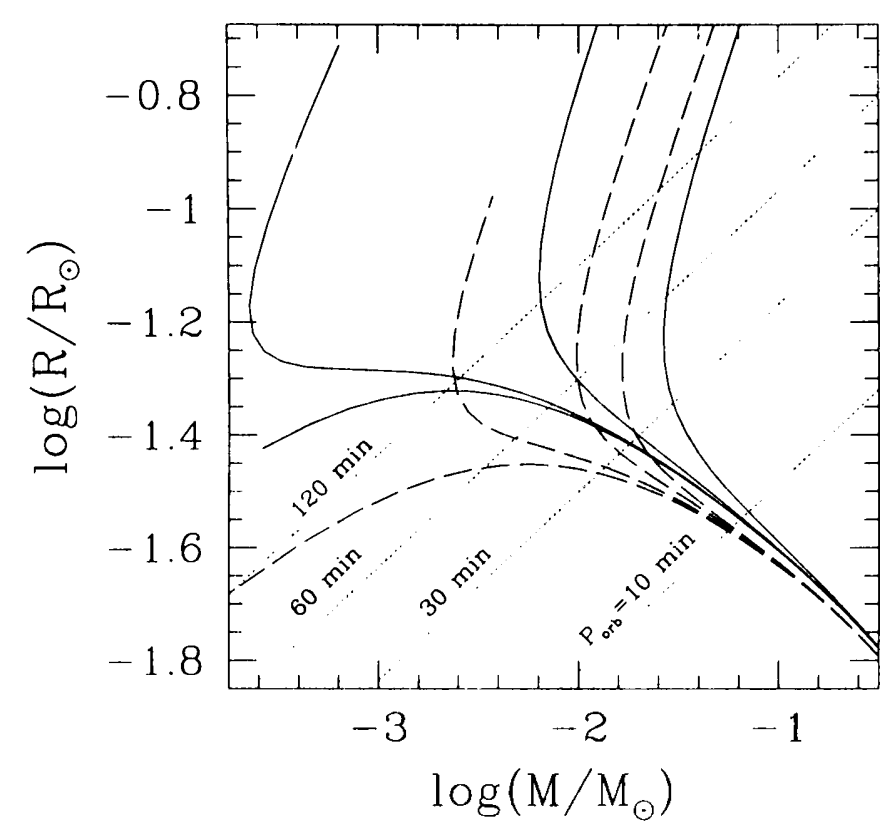

Fig. 1. The $M /-R$ relations along lines of constant $T_{c}$ for $\mathrm{He}\left(T_{r}=10^{4}, 10^{5}, 10^{6}, \& 5 \times 10^{6} \mathrm{~K}\right)$ and $\mathrm{C}\left(T_{c}=\right.$ $\left.10^{4}, 10^{6}, 3 \times 10^{6}, \& 5 \times 10^{6} \mathrm{~K}\right)$ donors.

reached if donors are either heated during their evolution (via tidal mechanisms?) or if they start mass transfer as hot objects at longer $P_{\text {orl }}$ (Nelson \& Rappaport 2003).

Differences in composition and $T_{r}$. make a donor more or less compact, altering the $P_{\text {orb }}$ at which the donor fills its RL and the resulting $M$. In addition, these variation also affect the rate at which the donor's radius, $R_{2}$, changes with mass loss, affecting the rate the system evolves through $P_{\text {orb }}$. The net effect is that $\mathrm{C} / \mathrm{O}$ donors evolve faster in $M_{2}$, but slower in $P_{\text {orb }}$ than He donors; hotter donors evolve faster in $P_{\text {orb }}$ than colder ones. Integrated over the population of UCBBs, these effects will alter our expectation for such things as the number distribution of UCBs with $P_{\text {orb }}$ (Deloye \& Bildsten 2003).

\section{REFERENCES}

Bildsten, L. 2002, ApJ, 577, L27

Deloye, C. J. \& Bildsten, L., 2003, ApJ, in press.

Fedorova, A. V. \& Ergma, E. V. 1989, Ap\&SS, 151, 125

Galloway, D. K., Chakrabarty, D., Morgan, E. H., Remil-

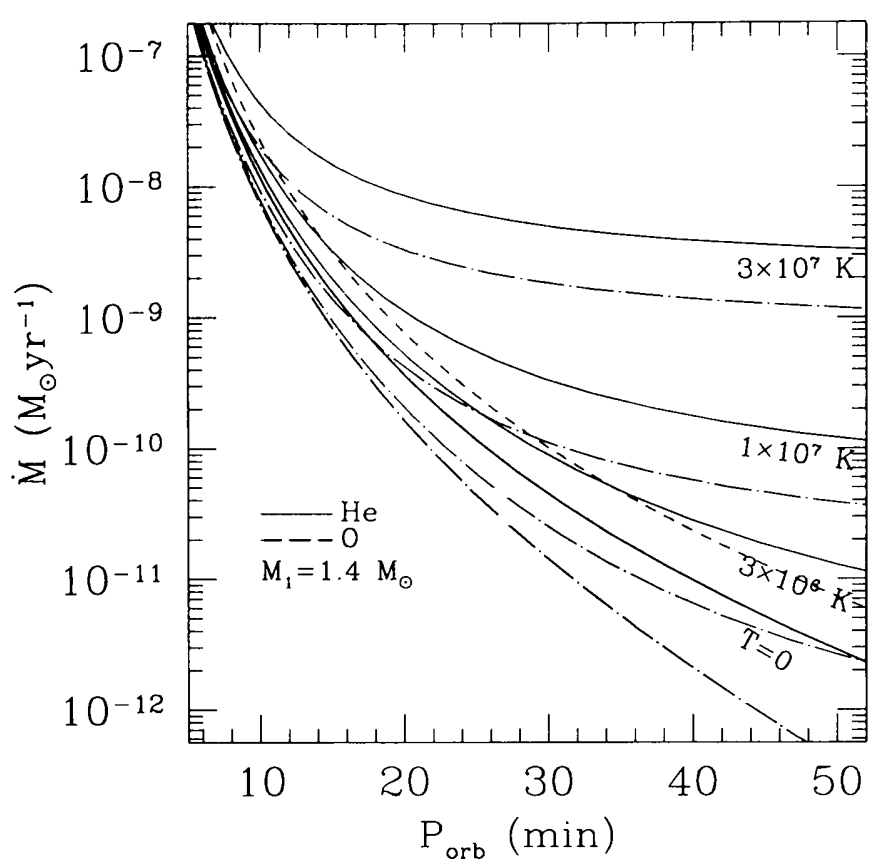

Fig. 2. How the $\dot{M}-P_{\text {mathrmorb }}$ relation is affected by the donor's $T_{c}$ and composition. The dashed line shows an adiabatic evolutionary track, highlighting that finding arbitrarily hot donors at longer $P_{\text {orb }}$ requires heating of the donor if the system evolved from contact at $P_{\text {orb }}<10$ $\min$.

lard, R. A. 2002, ApJ, 576, L137

Markwardt, C. B., Swank, J. H., Strohymayer, T. E., in 't Zand, J. J. M., \& Marshall, F. E. 2002, ApJ, 575, L21

Markwardt, C. B., Smith, E., \& Swank, J. H., 2003a, IAUC Circ. No. 8080

Markwardt, C. B., Juda, M., \& Swank, J. H., 2003b, IAUC Circ. No. 8095

Nelson, L. A., Rappaport, S. A., Joss, P. C. 1986, ApJ, 304,231

Nelson, L. A. \& Rappaport, S. A., 2003, ApJ, submitted, astro-ph/0304374

Podsiadlowski, Ph., Rappaport, S., \& Pfahl, E. D. 2002, ApJ, 565, 1107

Rappaport, S., Joss, P.C., \& Webbink, R. F. 1982, ApJ, 254,616

Salaris, M., García-Berro, E., Hernanz, M., Isern, J., \& Saumon, D. 2000, ApJ, 544, 1036

Serenelli, A. M., Althaus, L. G., Rohrmann, R. D., \& Benvenuto, O. G. 2001, MNRAS, 325, 607

C. J. Deloye: Dept. of Physics, Broida Hall, University of California, Santa Barbara, CA 93106 (cjdeloye@physics.ucsb.edu)

L. Bildsten: Kavli Institute for Theoretical Physics, Kohn Hall. University of California, Santa Barbara, CA 93106 (bildsten@kitp.ucsb.edu) 